\title{
LOSOM: phase relief imaging can be achieved with confocal system
}

Tong Peng, Hao Xie, Yichen Ding, Yiqing Lu, Dayong Jin, et al.

Tong Peng, Hao Xie, Yichen Ding, Yiqing Lu, Dayong Jin, Peng Xi, "LOSOM: phase relief imaging can be achieved with confocal system," Proc. SPIE 8553, Optics in Health Care and Biomedical Optics V, 85531C (11 December 2012); doi: 10.1117/12.2000809

SPIE. Event: Photonics Asia, 2012, Beijing, China 


\title{
LOSOM: Phase Relief Imaging Can Be Achieved with Confocal
}

\author{
System \\ Tong Peng ${ }^{1,2}$, Hao Xie ${ }^{1}$, Yichen Ding ${ }^{1}$, Yiqing $\mathrm{Lu}^{3}$, Dayong $\mathrm{Jin}^{3}$, and Peng Xi ${ }^{* 1}$ \\ ${ }^{1}$ Department of Biomedical Engineering, College of Engineering, Peking University, No. 5 \\ Yiheyuan Road, Beijing 100871, China \\ ${ }^{2}$ Department of Physics, Xi' an University of Technology, No. 5 South Jinhua Road, Xi' an \\ 710048, China \\ ${ }^{3}$ Advanced Cytometry Labs, MQphotonics Research Centre, Macquarie University, NSW 2109, \\ Sydney, Australia
}

\begin{abstract}
We reported recently that laser oblique scanning optical microscopy (LOSOM) is able to obtain a relief image in transparent sample directly. To optimize the performance of LOSOM, the parameters such as numerical aperture, the distance between the specimen and the fluorescent medium and the pinhole size are investigated in this work. A beam blocker is introduced in light path which enhances dramatically the visualization of local phase difference.
\end{abstract}

Key words: phase relief imaging; scanning optical microscopy; numerical aperture

\section{INTRODUCTION}

Due to its non-invasive and 3-D optical sectioning capability, confocal laser scanning microscopy (CLSM) has been widely applied in both biological research and clinical diagnosis in the last few decades. As an important parameter, optical phase can provide additional structural information of the transparent specimen. The request of obvserving optical phase has driven a variety of phase sensing microscopy available, such as phase contrast (PhC)[1-3] microscopy and differential interference contrast (DIC) microscopy[4-6]. Both of these techniques rely on the fact that the incident light pass through a transparent specimen with different optical length, which leads to a local phase-modulated intensity.

Previously, we have presented a novel phase sensitive imaging technique named laser oblique scanning optical microscope (LOSOM), which is based on a CLSM with the introduction of a fluorescent medium[7]. Through this novel method, both confocal fluorescent image and a similar 3D phase-relief LOSOM image can be acquired simultaneously.

In this paper, we further demonstrated the advantages and features of LOSOM microscopy, such as its capability of working with different confocal parameters (for example, the different numerical aperture (NA) pinhole size, and the signal intensity with different distance of the specimen from the fluorescent medium. We further demonstrate that in LOSOM, the parameters can be optimized to enhance the performance of imaging specimen with 3-D phase relief effect. The presented work could dramatically enhance the application of LOSOM phase-relief imaging technique in a variety of biological application[4, 8-9].

Optics in Health Care and Biomedical Optics V, edited by Qingming Luo, Ying Gu, Xingde D. Li,

Proc. of SPIE Vol. 8553, 85531C · C 2012 SPIE · CCC code: 1605-742/12/\$18

doi: $10.1117 / 12.2000809$

Proc. of SPIE Vol. 8553 85531C-1 


\section{METHOD}

\subsection{Setup}

In this paper, the LOSOM system was built and the schematic diagram of the experimental setup was depicted in Fig 1.The $375 \mathrm{~nm}$ ultra-violet light (FC-375-20-SM-PC-0-0, RGBLase LLC, USA)was used for light source. The excitation light passed through the dichroic mirror(DM, Z380trans-DC-XR,USA), arrived at the galvonometer for raster scanning, and then passed the scan lens, tube lens and a 10x objective lens (PlanFluor NA 0.3, Nikon, Japan), and then focused on the sample successively. The exciting fluorescent would go back to the DM and focused on the fiber collimators (F220FC-A, Thorlabs) for fiber coupling. A 50 um multi-mode fiber (M1-F8 F8-07-0010-GF, Go4Fibers, Hong Kong) used also served as the pinhole for confocal fluorescence collection.

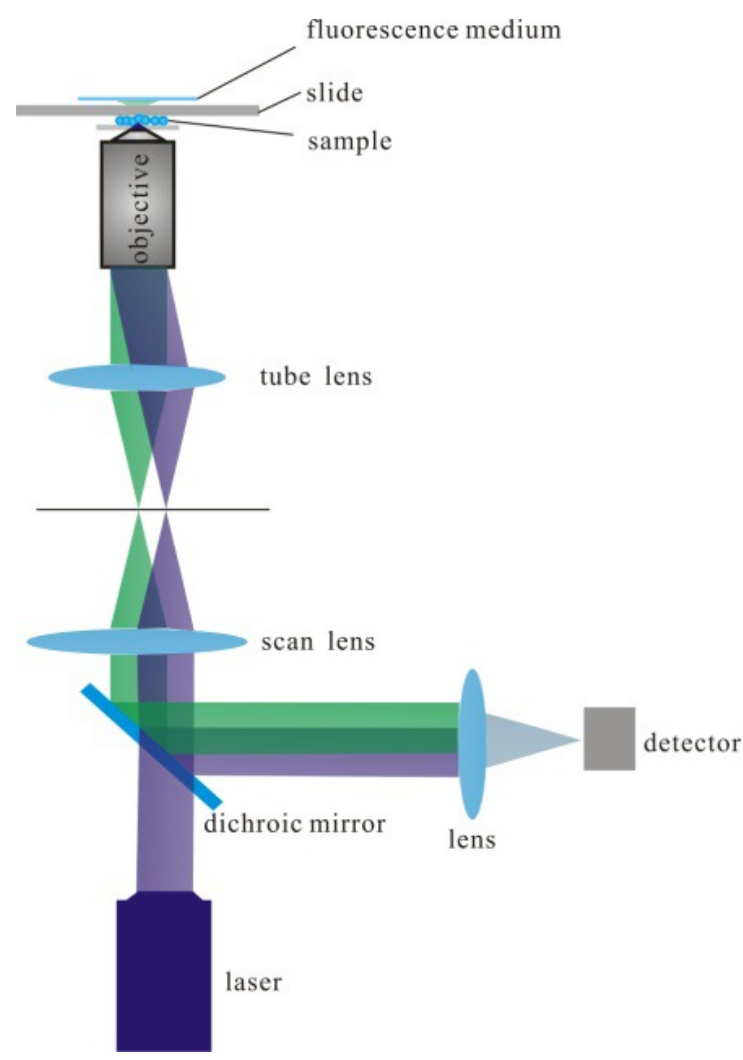

Fig.1The schematic diagram of the LOSOM system

\subsection{Parameters of confocal system setup for LOSOM phase-relief imaging}

\subsubsection{Numerical aperture}

As an important parameter of LOSOM, numerical aperture (NA) was related to the imaging quality directly. Previously, we have demonstrated that LOSOM is compatible with at least $\mathrm{NA}<0.5$, to evaluate the influence of the imaging result with different NA, the varied objective lens were used for comparison and the results were showed in fig.2.

Fig 2 illustrated the result with different NA objective lens, from (a) (c), the NA was 0.3 (10x), 0.45 (20x) and 1.3 (40x) respectively. As can be seen from Fig. 2 that, the higher the numerical aperture, the larger the fluorescent medium being 
excited, and consequently the lower the detected signal is. By increasing the gain of the PMT, LOSOM with 40x NA 1.3 objective has been successfully demonstrated. As we know, the resolution of the confocal system can be expressed as:

$$
d=\frac{\lambda}{2 \sqrt{2} N A}
$$

where $\lambda$ denotes the wavelength of laser and NA, the numerical aperture of the objective lens. With this expression, the higher the NA of the objective, the better of the resolution can achieve. Our experimental results demonstrated well accordance with this.
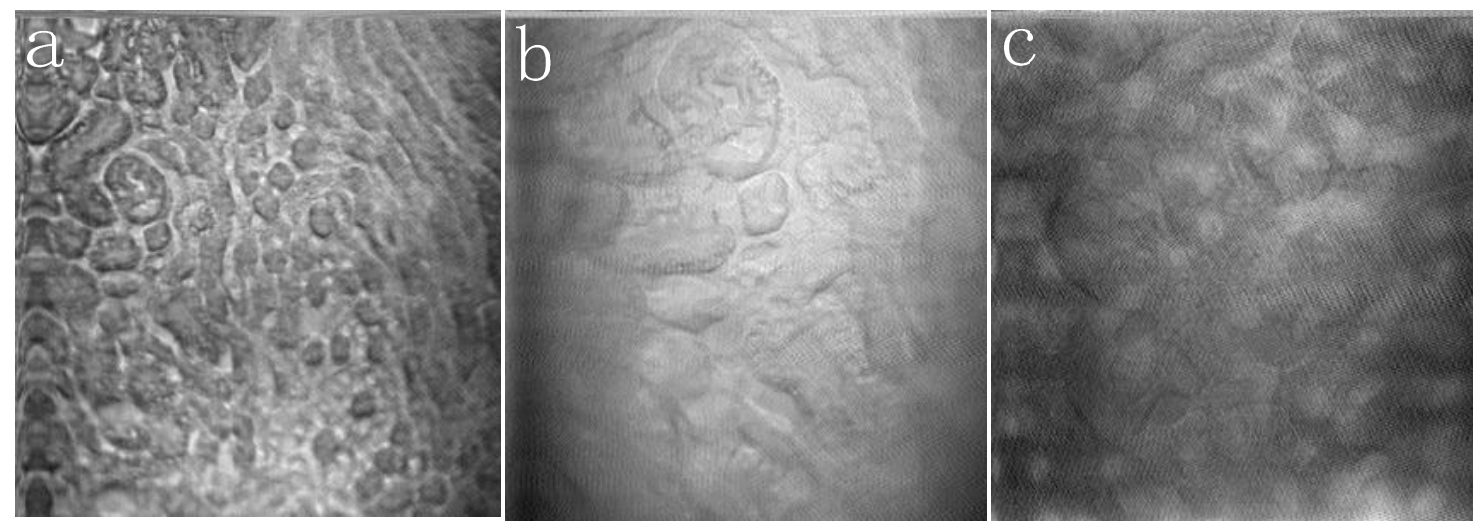

Fig.2 Mouse kidney imaged results with different objective. (a)10x;(b)20x;(c)40x

\subsubsection{Distance between the specimen and the fluorescent medium}

The distance between the specimen and fluorescent medium has been set to $1.2 \mathrm{~mm}$, which is the thickness of the slide glass. The principle of LOSOM reveals that the distance between the sample and the fluorescent medium well largely affect the signal intensity detected.
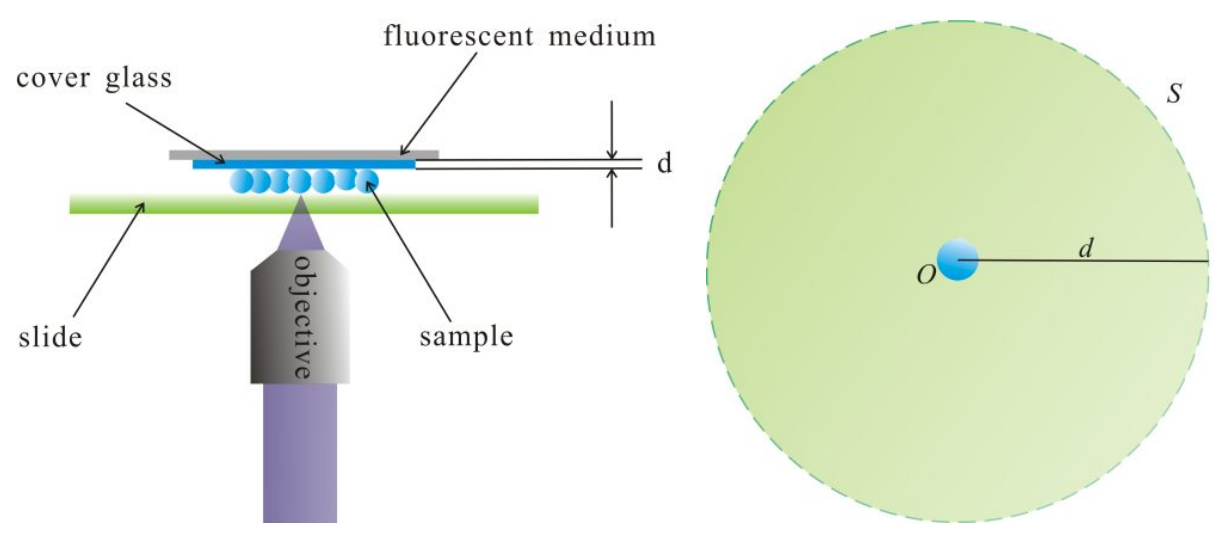

Fig 3 Schematic diagram of the specimen part of the LOSOM.

Fig.3 illustrated part of the LOSOM system, where the distance between the sample and the fluorescent medium was $d$. The intensity of the point in spherical is inversely proportional with the spherical area. Thus, the inverse of square of d, since the fluorescence emitted by the sample was spherical wave with isotropic properties (Fig. 3(b)). 
Therefore, decreasing $\mathrm{d}$ can increase the signal level for LOSOM phase-relief imaging. To study this effect quantitatively, we have designed a special piece of glass slide, which is composed of multiple hollow coverglasses, each with thickness of $0.17 \mathrm{~mm}$. The imaged result and the curve of selected area intensity verse different $d$ was showed in fig.4. Due to our hypothesis, the intensity was inverse of square of $d$, and the relationship of the intensity and d was plotted in fig.4 (b), from which, the multinomial fitting used here was expressed as

$$
I=\mathrm{a} \cdot d^{b}+c
$$

Where $\mathrm{a}, \mathrm{b}$ and $\mathrm{c}$ were the coefficient fitted. From the fitted result, we get $\mathrm{a}=1209, \mathrm{~b}=-2.257$ and $\mathrm{c}=56.98$, which were according with the hypothesis.
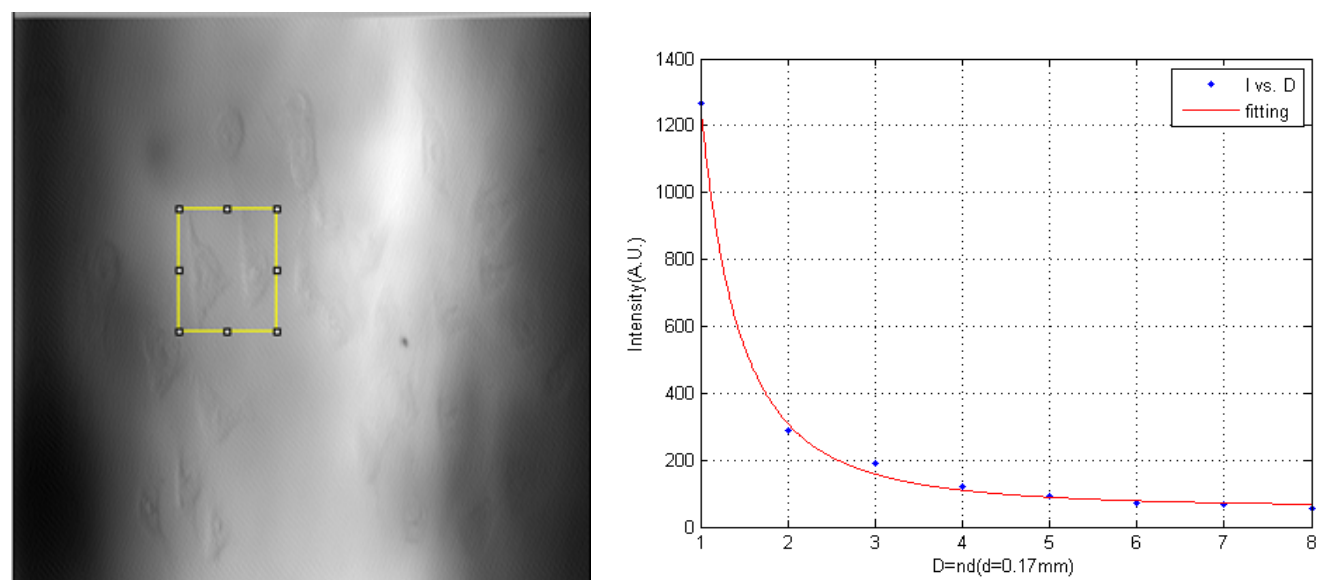

Fig 4.the relationship of the intensity and d

\subsubsection{Pinhole size}

Like confocal microscopy, LOSOM used a pinhole to prevent fluorescence out of focus and enhancement of the contrast of image. The pinhole size also was a determining factor of LOSOM and limitation of the signal to noise ratio (SNR) of the final result. A 5AU pinhole has been used previously. In order to demonstrate that the LOSOM phase relief image was not a result of large pinhole, we have tested pinhole size of 1AU, and 5AU respectively. Fig5 illustrated the result with different $\mathrm{AU}$, as could be seen from the figure that, the fringe of the tissue was modulated only by the oblique angle, and had no effect on the pinhole size.
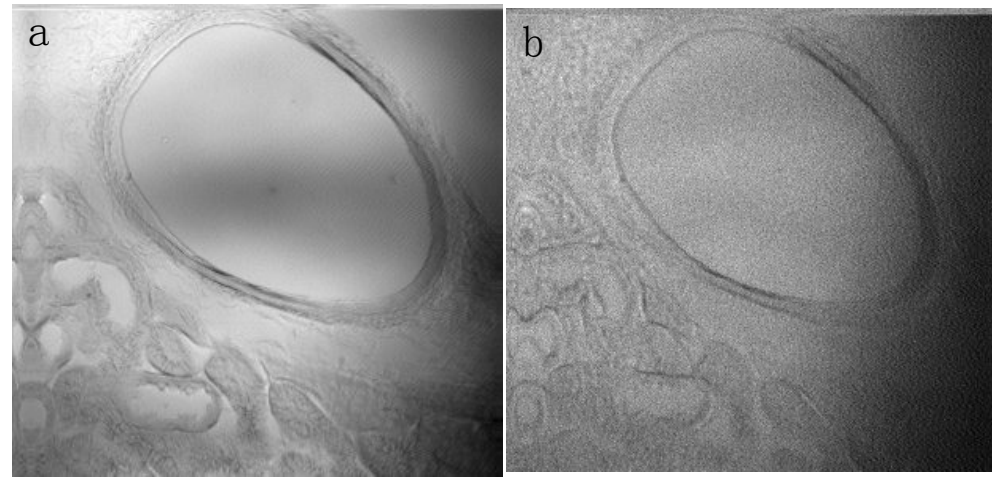

Fig. 5 LOSOM with different AU. (a) $5 \mathrm{AU}$ and (b)1 AU 


\subsubsection{Obstruction}

In a typical wide-field microscopy, according to the mutual coherence transport equation[10],

$$
J_{1}^{i n}\left(x_{1}, x_{1}^{\prime}\right)=\int_{\alpha_{1}}^{\alpha_{2}} d \xi_{0} \int_{\alpha_{1}}^{\alpha_{2}} d \xi_{0}^{\prime} J_{0}\left(\xi_{0}, \xi_{0}^{\prime}\right) e^{-i\left(x_{1} \xi_{0}-x_{1}^{\prime} \xi_{0}^{\prime}\right)}
$$

Light intensity at detector can be derived as:

$$
I_{3}\left(x_{3}\right)=\iint\left(K_{e}\left(x_{3}+x_{1 c}, x_{1 d}\right) T_{r}\left(x_{1 c}, x_{1 d}\right)+K_{o}\left(x_{3}+x_{1 c}, x_{1 d}\right) T_{i}\left(x_{1 c}, x_{1 d}\right)\right) d x_{1 c} d x_{1 d}
$$

That means Ke and Ko serve as filters for real and image parts of transmission rate respectively. Numerical calculations indicate whether there is a blocker in light path, making local phase differences reflected in image intensity.

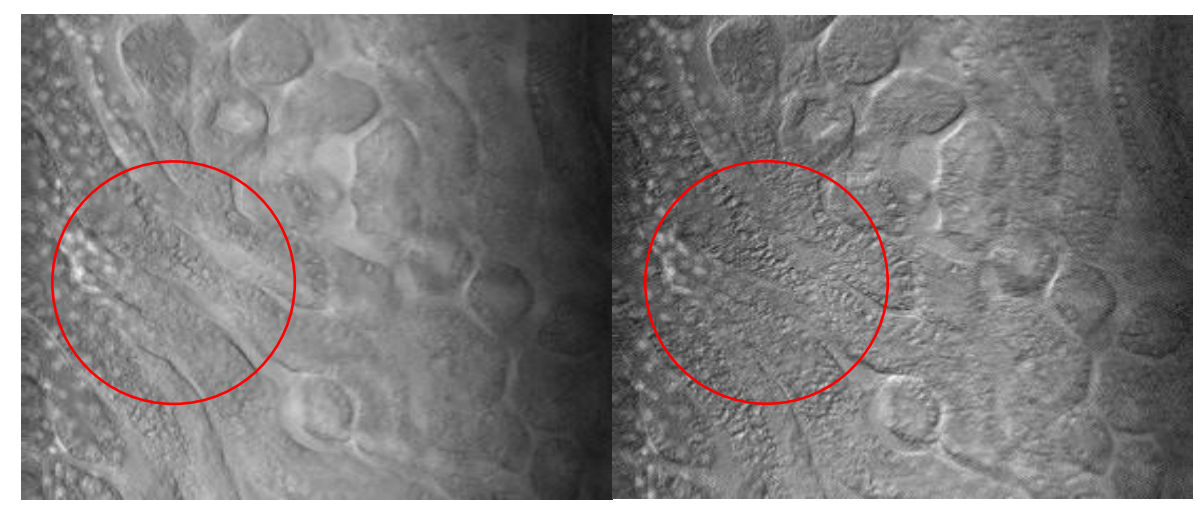

Fig. 6 comparison of unobstruct (a) and obstruct (b)

As it could be seen clearly in fig. 6 that with part of the light obstructed, the phase relief effect was more obvious and information was more visualized. The red circle regions representing the different of the two results. As can be seen, although the intensity of image area is decreased with obstruction, the 3-D relief effect became more obvious, and more details of the tissue were revealed.

\section{CONCLUSION}

Based on confocal laser scanning microscopy, by introducing an addition fluorescence medium, the LOSOM system was achieved and 3-D phase relief image was realized, which provided a novel imaging method with phase sensitivity. In order to optimize the performance of LOSOM, parameters such as numerical aperture, the distance between the specimen and the fluorescent medium and the pinhole size were investigated. With theoretical analysis, the numerical calculations indicated that the presence of an obstructor in light pathcan enhance the local phase-relief result.

\section{ACKNOWLEDGMENT}

This research is supported by the National Natural Science Foundation of China (61178076), Shanghai Municiple Commission of Science and Technology (10220706900), and seed fund support from Olympus Australia. 


\section{REFERENCES}

[1] Burch, C. and J. Stock, Phase-contrast microscopy. Journal of Scientific Instruments, 2002. 19(5): p. 71.

[2] Zernike, F., Phase contrast, a new method for the microscopic observation of transparent objects. Physica, 1942. 9: p. 686-698.

[3] Zernike, F., Phase contrast, a new method for the microscopic observation of transparent objects Part II. Physica, 1942. 9(10): p. 974-986.

[4] Arnison, M.R., et al., Linear phase imaging using differential interference contrast microscopy. Journal of microscopy, 2004. 214(1): p. 7-12.

[5] Nomarski, G., Differential microinterferometer with polarized waves. J. Phys. Radium, 1955. 16(9).

[6] Preza, C., D.L. Snyder, and J.A. Conchello, Theoretical development and experimental evaluation of imaging models for differential-interference-contrast microscopy. JOSA A, 1999. 16(9): p. 2185-2199.

[7] Ding, Y., et al., Laser oblique scanning optical microscopy (LOSOM) for phase relief imaging. Optics Express, 2012. 20(13): p. 14100-14108.

[8] Allen, R.D., N.S. Allen, and J.L. Travis, Video - enhanced contrast, differential interference contrast (AVEC - DIC) microscopy: A new method capable of analyzing microtubule - related motility in the reticulopodial network of allogromia laticollaris. Cell motility, 1981. 1(3): p. 291-302.

[9] Nagayama, K. and R. Danev, Phase contrast electron microscopy: development of thin-film phase plates and biological applications. Philosophical Transactions of the Royal Society B: Biological Sciences, 2008. 363(1500): p. 2153-2162.

[10] Yi, R., K.K. Chu, and J. Mertz, Graded-field microscopy with white light. Optics Express, 2006. 14(12): p. 5191-5200. 